\title{
Estimating baseline creatinine values to define acute kidney injury in critically ill pediatric patients
}

\author{
Yeon Joo Lee, Young Seo Park, Seong Jong Park, Won Kyoung Jhang \\ Department of Pediatrics, Asan Medical Center Children's Hospital, University of Ulsan College of Medicine, Seoul, Republic of Korea
}

\begin{abstract}
Background: Acute kidney injury (AKI) is a common complication in critically ill children. However, the common lack of baseline serum creatinine values affects AKI diagnosis and staging. Several approaches for estimating baseline creatinine values in those patients were evaluated.

Methods: This single-center retrospective study enrolled pediatric patients with documented serum creatinine measurements within 3 months before admission and more than two serum creatinine measurements within 7 days after admission to the pediatric intensive care unit of a tertiary care children's hospital between January 2016 and April 2020. Four different approaches for estimating AKI using serum creatinine measurements were compared: 1) back-calculation using age-adjusted normal reference glomerular filtration rates, 2) age-adjusted normal reference serum creatinine values, 3) minimum values measured within 7 days after admission, and 4) initial values upon admission.

Results: The approach using minimum values showed the best agreement with the measured baseline value, with the largest intraclass correlation coefficient (0.623), smallest bias (-0.04), and narrowest limit of agreement interval (1.032). For AKI diagnosis and staging, the minimum values were $80.8 \%$ and $76.1 \%$ accurate, respectively. The other estimated baseline values underestimated AKI and showed poor agreement with baseline values before admission, with a misclassification rate of up to $42 \%(p<0.001)$.

Conclusion: Minimum values of serum creatinine measured within 7 days after hospital admission showed the best agreement with creatinine measured within 3 months before admission, indicating the possibility of using it as a baseline when baseline data are unavailable. Further large-scale studies are required to accurately diagnose AKI in critically ill children.
\end{abstract}

Keywords: Acute kidney injury, Creatinine, Critical illness, Pediatrics

\section{Introduction}

Acute kidney injury (AKI) is a common problem associated with increased adverse outcomes and high mortality in critically ill pediatric patients [1-4]. AKI in children is currently diagnosed using standardized diagnostic criteria derived from those for adults [5-10], most of which use changes in baseline serum creatinine values (SCr-base) or the glomerular filtration rate (GFR) for diagnosing and staging AKI. Ideally, SCr-base accurately reflects a patient's steadystate kidney function before the onset of AKI [11]. However, many patients do not have preadmission SCr-base values in

Received: May 29, 2021; Revised: August 27, 2021; Accepted: September 10, 2021

Correspondence: Won Kyoung Jhang

Division of Pediatric Critical Care Medicine, Department of Pediatrics, Asan Medical Center Children's Hospital, University of Ulsan College of

Medicine, 88 Olympic-ro 43-gil, Songpa-gu, Seoul 05505, Republic of Korea. E-mail: wkjhang@amc.seoul.kr

ORCID: https://orcid.org/0000-0003-2309-0494

Copyright (C) 2022 by The Korean Society of Nephrology

(a) This is an Open Access article distributed under the terms of the Creative Commons Attribution Non-Commercial and No Derivatives License (http:// creativecommons.org/licenses/by-nc-nd/4.0/) which permits unrestricted non-commercial use, distribution of the material without any modifications, and reproduction in any medium, provided the original works properly cited. 
their records, which complicates AKI diagnosis and staging [12-14].

In the absence of consensus-based standardized methods for replacing missing SCr-base data, several values have been suggested for estimating a surrogate SCr-base value, including the initial admission $\mathrm{SCr}$ values, minimum SCr values during hospitalization, dynamic SCr values during a 7-day or 48-hour window, or back-calculation by imputing an estimated GFR (eGFR) of $75 \mathrm{~mL} / \mathrm{min} / 1.73 \mathrm{~m}^{2}$ with the Modification of Diet in Renal Disease (MDRD) equation or the Chronic Kidney Disease Epidemiology Collaboration formula [13,15-20]. However, those substitutes have mainly been studied and validated in adult populations. Few studies have evaluated the various estimation approaches in critically ill children. Therefore, it is difficult to determine which SCr-base surrogate should be used to evaluate AKI and related clinical outcomes in critically ill pediatric patients.

This study evaluated several approaches for calculating an estimated SCr-base (eSCr-base) value, including methods commonly used in adult patients and those specified and adjusted for use in pediatric patients. The eSCr-base values calculated using different approaches were compared with a reference SCr-base value measured 3 months or less before admission (mSCr-base). The value of the different estimations in diagnosing and staging AKI was also compared.

\section{Methods}

\section{Study subjects}

This study was a single-center, retrospective cohort study. All critically ill children consecutively admitted to a 14bed multidisciplinary pediatric intensive care unit (PICU) in Asan Medical Center Children's Hospital, Seoul, Korea between January 2016 and April 2020 were screened. Patients aged 1 month to 18 years with available mSCr-base data, defined as the lowest value measured 3 months or less before PICU admission, and at least two documented SCr measurements within the first 7 days of PICU admission were enrolled. Exclusion criteria were as follows: age of $<1$ month or $>18$ years, no available mSCr-base value, known kidney anomalies, preexisting chronic renal failure, dialysis used before PICU admission, PICU stay of $<24$ hours, and do-not-resuscitate orders (Fig. 1).

This study was approved by the Institutional Review Board of Asan Medical Center (No. 2020-0878), which waived the requirement for informed consent because the study was retrospective. This study was conducted in accordance with the principles of the 1964 Declaration of Helsin-

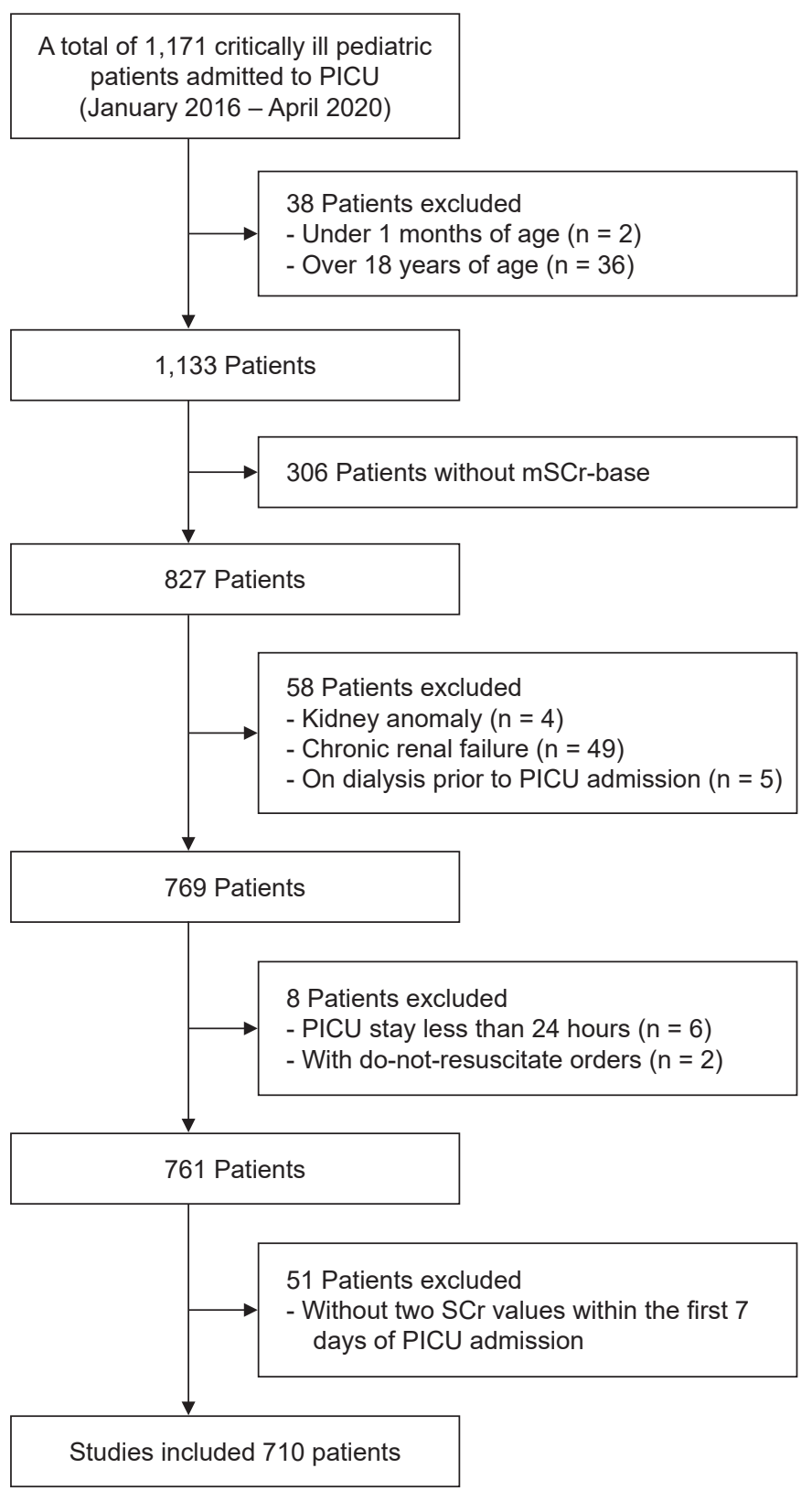

Figure 1. Flow chart for inclusion and exclusion of the study population.

mSCr-base, measured SCr-base (within 3 months prior to admission); PICU, pediatric intensive care unit; SCr, serum creatinine. 
ki and later amendments. It also conforms to the Strengthening the Reporting of Observational Guidelines statement for reporting observational studies.

\section{Data collection}

We retrospectively reviewed the electronic medical records of all included patients and collected data on their baseline demographic characteristics, underlying disorders, reasons for PICU admission, initiation of continuous renal replacement therapy (CRRT), duration of PICU stay, 28-day mortality rate, and laboratory findings, including SCr values. We evaluated four different methods for calculating an eSCr-base value: 1) back-calculation of SCr values using the age-adjusted normal reference value for eGFR (SCr-eGFR) [21-23] (Supplementary Table 1, available online 2) normal reference values adopted from the pediatric Sequential Organ Dysfunction Assessment (pSOFA) (SCr-ref) [24] (Supplementary Table 1); 3) minimum SCr value measured within 7 days after PICU admission (SCr-min); and 4) initial SCr values measured at PICU admission (SCr-adm). Imputation of SCr-base by back-calculation was performed using the original Schwartz formula because it was derived from SCr measured using the Jaffe method, which is used in our institution [22,25-28]. To evaluate disease severity and organ dysfunction, the Pediatric Risk of Mortality III and pSOFA scores were calculated using the worst documented values within the first 24 hours of PICU admission [24,29].

\section{Study design}

The primary outcome was agreement between mSCr-base and the eSCr-base values calculated using the different methods. For this purpose, reliability analyses were performed to assess the intraclass correlation coefficients (ICCs). In addition, descriptive statistics were used to calculate the bias (mean difference) and limit of agreement (LOA), and the results are depicted as scatterplots. The secondary outcome was the performance of the various eSCr-base values in AKI diagnosis and staging. For that purpose, we compared the AKI diagnoses and stages determined using the various eSCr-base values with those made using mSCr-base.

\section{Definitions}

AKI was defined as an absolute increase in $\mathrm{SCr}$ of $0.3 \mathrm{mg} /$ $\mathrm{dL}$ within 48 hours or a $>50 \%$ relative increase in $\mathrm{SCr}$ compared with "SCr-base" during the first 7 days of PICU admission (higher than stage 1 of the Kidney Disease: Improving Global Outcomes [KDIGO] 2012 criteria) [10]. AKI was diagnosed and classified according to the definition of the KDIGO workgroup, which considers the maximum increase in SCr during the first 7 days of PICU admission. The urine output criteria were not used because of data uncertainty.

\section{Statistical analyses}

The data were analyzed using IBM SPSS for Windows, version 21.0 (IBM Corp., Armonk, NY, USA). Continuous variables with a normal distribution are reported as means and standard deviations (SDs), and those with skewed distributions are reported as medians and interquartile ranges (IQRs). Normality was determined using the Kolmogorov-Smirnov test. Categorical variables are expressed as numbers and proportions. The agreement between eSCr-base and mSCr-base was assessed using a reliability analysis with ICCs. The bias and LOA (defined as the bias $\pm 1.96 \times \mathrm{SD}$ ), were calculated using descriptive statistics. The performance of the various eSCr-base values in AKI diagnosis and staging was compared with the results with mSCr-base using chi-square testing in a trend analysis. Based on crosstables of the mSCr-base and eSCr-base results, we calculated and report the sensitivity, specificity, positive predictive value $(\mathrm{PPV}=$ true positive $/[$ true positive + false positive]), negative predictive value (NPV $=$ true negative/[true negative + false negative]), positive likelihood ratio (PLR = sensitivity/1 - specificity), negative likelihood ratio (NLR = 1 - sensitivity/specificity), misclassification rate, and percent positive agreement. Kappa statistics with $95 \%$ confidence intervals (CIs) are used to report the agreement levels. The misclassification rate was evaluated using McNemar test. For all analyses, variables with a two-sided $\mathrm{p}$-value of $<0.05$ were considered statistically significant. 
Table 1. Baseline characteristics of the study population

\begin{tabular}{|c|c|}
\hline Variable & Data \\
\hline No. of patients & 710 \\
\hline Male sex & $383(53.9)$ \\
\hline Age (mo) & $23.0(7.0-88.0)$ \\
\hline Weight (kg) & $10.0(5.7-21.0)$ \\
\hline Height (cm) & $81.0(63.0-118.2)$ \\
\hline Duration of PICU stay (day) & $7(3-16)$ \\
\hline Hospital stay (day) & $37(19-82)$ \\
\hline \multicolumn{2}{|l|}{ Underlying disease } \\
\hline Cardiac & $200(28.2)$ \\
\hline Hemato-oncologic & $158(22.3)$ \\
\hline Gastrointestinal/hepatic & $121(17.1)$ \\
\hline Respiratory & $83(11.7)$ \\
\hline Neurologic & $49(6.9)$ \\
\hline Genetic & $44(6.2)$ \\
\hline Endocrinologic & $24(3.4)$ \\
\hline Nephrology & $11(1.6)$ \\
\hline None & $19(2.7)$ \\
\hline \multicolumn{2}{|l|}{ Cause of PICU admission } \\
\hline Respiratory problems & $281(39.6)$ \\
\hline Gastrointestinal/hepatic problems & $133(18.7)$ \\
\hline Cardiac problems & $103(14.5)$ \\
\hline Shock & $64(9.0)$ \\
\hline Neurological problems & $48(6.8)$ \\
\hline Hemato-oncologic problems & $30(4.2)$ \\
\hline Nephrological problems & $24(3.4)$ \\
\hline Post-cardiopulmonary arrest & $12(1.7)$ \\
\hline Others & $15(2.1)$ \\
\hline CRRT within 7 days of PICU admission & $48(6.8)$ \\
\hline Presence of $\mathrm{AKI}$ & $417(58.7)$ \\
\hline Stage 1 & $166(39.8)$ \\
\hline Stage 2 & $112(26.9)$ \\
\hline Stage 3 & $139(33.3)$ \\
\hline Malnutrition & $192(27.0)$ \\
\hline 28-Day mortality & $49(6.9)$ \\
\hline mSCr-base & $0.19(0.17-0.31)$ \\
\hline SCr-eGFR & $0.49(0.40-0.66)$ \\
\hline SCr-ref & $0.40(0.30-0.70)$ \\
\hline SCr-min & $0.21(0.17-0.34)$ \\
\hline SCr-adm & $0.32(0.22-0.53)$ \\
\hline PRISM III score & $9.0(5.3-14.0)$ \\
\hline pSOFA score & $6.0(4.0-8.0)$ \\
\hline
\end{tabular}

Data are expressed as number only, number (\%), or median (interquartile range).

AKI, acute kidney injury; CRRT, continuous renal replacement therapy; eGFR, estimated glomerular filtration rate; mSCr-base, measured SCrbase (within 3 months prior to admission); PICU, pediatric intensive care unit; PRISM, Pediatric Risk of Mortality; pSOFA, pediatric Sequential Organ Failure Assessment; $\mathrm{SCr}$, serum creatinine; $\mathrm{SCr}$-adm, initial $\mathrm{SCr}$ value measured at PICU admission; SCr-eGFR, back-calculation of $\mathrm{SCr}$ values using the age-adjusted normal reference value of eGFR; SCr-min, minimum SCr value measured within 7 days after PICU admission; SCr-ref, normal reference $\mathrm{SCr}$ values adopted from pSOFA.
Table 2. The ICCs, bias (mean difference), and LOAs between mSCr-base and SCr-eGFR, SCr-ref, SCr-min, and SCr-adm

\begin{tabular}{lccc}
\hline Estimation method & ICC & Bias & LOA \\
\hline SCr-eGFR & 0.35 & -0.27 & 1.13 \\
SCr-ref & 0.34 & -0.25 & 1.19 \\
SCr-min & 0.62 & -0.04 & 1.03 \\
SCr-adm & 0.57 & -0.22 & 1.68 \\
\hline
\end{tabular}

eGFR, estimated glomerular filtration rate; ICC, intraclass correlation coefficients; LOA, limits of agreement; SCr, serum creatinine; SCr-adm, initial $\mathrm{SCr}$ value measured at pediatric intensive care unit admission; SCr-eGFR, back-calculation of $\mathrm{SCr}$ values using age-adjusted normal reference value of eGFR; SCr-min, minimum SCr value measured within 7 days after pediatric intensive care unit admission; SCr-ref, normal reference $\mathrm{SCr}$ values adopted from the pediatric Sequential Organ Dysfunction Assessment score.

\section{Results}

\section{Baseline characteristics of the study population}

A total of 710 patients were included. The median (IQR) age and body weight were 23.0 months (7.0-88.0 months) and $10.0 \mathrm{~kg}(5.7-21.0 \mathrm{~kg})$, respectively. Cardiac $(28.2 \%)$ and hemato-oncological disorders $(22.3 \%)$ were the most frequently encountered underlying disorders. Respiratory problems (39.6\%) were the most common indication for PICU admission (Table 1). Forty-eight patients (6.8\%) required CRRT within the first 7 days of PICU admission. The overall 28 -day mortality rate was $6.9 \%$. Of all patients, $27 \%$ were malnourished.

\section{Comparison of $\mathrm{mSCr}$-base and eSCr-base}

The ICCs between mSCr-base and SCr-eGFR, SCr-ref, SCrmin, and SCr-adm were $0.351,0.337,0.623$, and 0.571 , respectively (Table 2). Fig. 2 shows the degree of agreement between mSCr-base and each eSCr-base value. According to the corresponding measurements of agreement, the largest bias was found between mSCr-base and SCr-eGFR $(-0.21)$. SCr-adm showed the worst agreement with mSCrbase and the widest LOA interval (1.683), whereas SCr-min showed the best agreement, with the smallest bias $(-0.04)$ and narrowest LOA interval (1.032) (Table 2). 

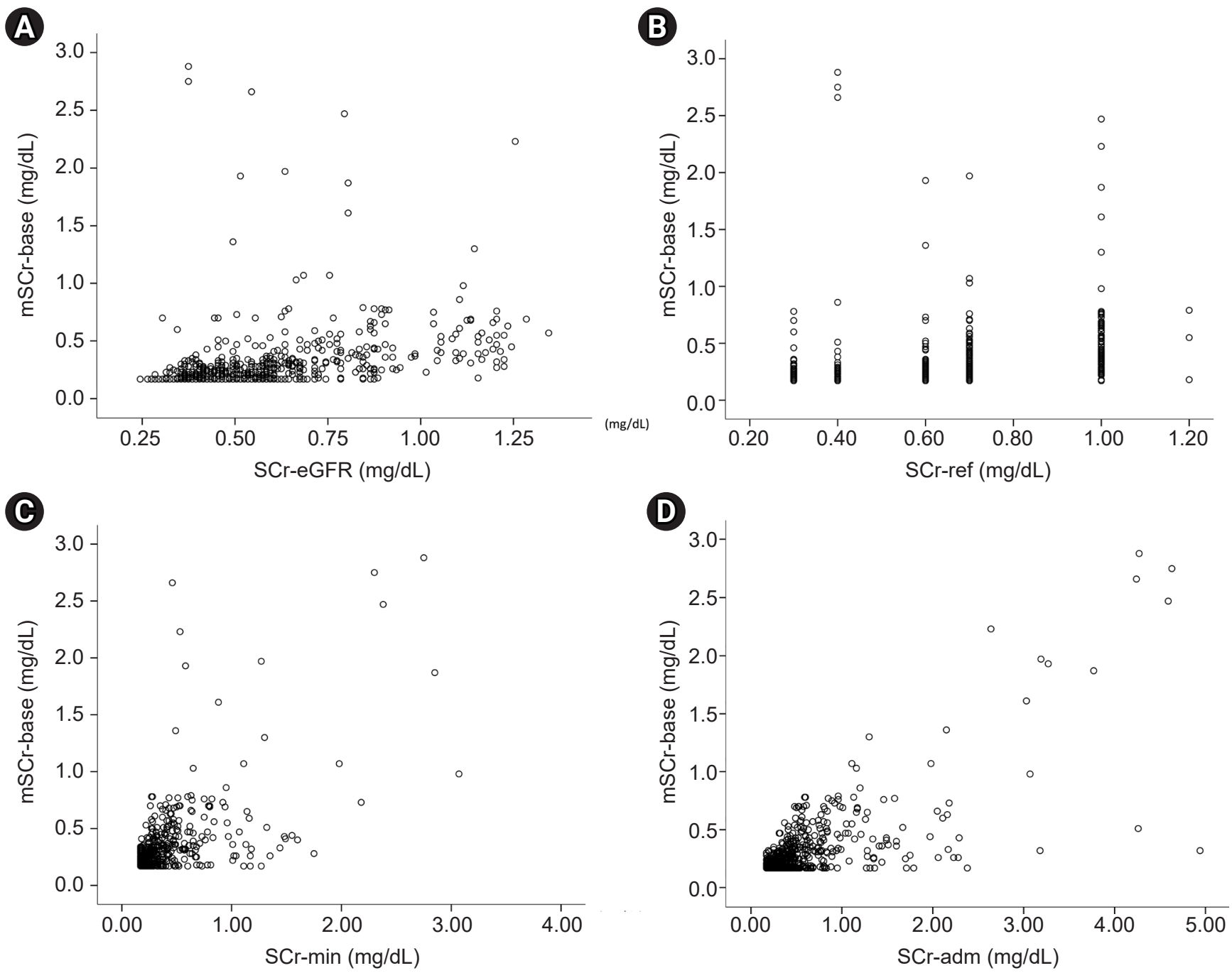

Figure 2. Scatterplots comparing mSCr-base and estimated SCr-base values derived using four different estimating approaches. (A) Back-calculation of SCr values using age-adjusted normal reference values for the eGFR (SCr-eGFR). (B) Normal reference SCr values adopted from the pediatric Sequential Organ Dysfunction Assessment score (SCr-ref). (C) Minimum SCr value measured within 7 days after PICU admission (SCr-min). (D) Initial SCr value at PICU admission (SCr-adm).

eGFR, estimated glomerular filtration rate; mSCr-base, measured SCr-base (within 3 months prior to admission); PICU, pediatric intensive care unit; SCr, serum creatinine.

Evaluation of estimated serum creatinine-based performance

\section{Acute kidney injury diagnosis based on different estimated} serum creatinine-based values

The incidence of AKI calculated using mSCr-base was $58.7 \%$, whereas it was overestimated as $63.5 \%$ by SCrmin. All other eSCr-base values (SCr-eGFR, SCr-ref, and SCr-adm) underestimated the incidence of AKI (as 19.1\%,
$22.1 \%$, and $23.8 \%$, respectively). Table 3 presents the sensitivity, specificity, PPV, NPV, PLR, NLR, misclassification rate, percent positive agreement, McNemar test results, and kappa statistics of the various eSCr-base values compared with mSCr-base. Among the eSCr-base values, SCr-min showed the best agreement for AKI diagnosis, with a positive percent agreement of $80.8 \%$ and kappa statistic of 0.598 (95\% CI, 0.537-0.659). 
Table 3. Performance of the tested estimation methods in diagnosing and staging AKI relative to the use of baseline SCr levels measured preadmission

\begin{tabular}{lccccccccccc}
\hline $\begin{array}{l}\text { Estimation } \\
\text { method }\end{array}$ & $\begin{array}{c}\text { Diagnosis } \\
\text { of AKI (\%) }\end{array}$ & $\begin{array}{c}\text { Sensitivity } \\
(\%)\end{array}$ & $\begin{array}{c}\text { Specificity } \\
(\%)\end{array}$ & PPV & NPV & PLR & NLR & $\begin{array}{c}\text { Misclassifica- } \\
\text { tion rate (\%) }\end{array}$ & $\begin{array}{c}\text { Percent } \\
\text { agreement } \\
(\%)\end{array}$ & $\begin{array}{c}\text { McNemar } \\
\text { test } \\
(p-v a l u e)\end{array}$ & $\begin{array}{c}\text { Kappa statistics } \\
(95 \% ~ C l)\end{array}$ \\
\hline mSCr-base & 58.7 & & & & & & & & & & \\
SCr-eGFR & 19.1 & 31.5 & 98.3 & 0.962 & 0.506 & 18.437 & 0.697 & 40.3 & 58.7 & $<0.001$ & $0.26(0.22-0.31)$ \\
SCr-ref & 22.1 & 36.5 & 98.3 & 0.968 & 0.521 & 21.360 & 0.647 & 38.0 & 62.0 & $<0.001$ & $0.31(0.26-0.36)$ \\
SCr-min & 63.5 & 87.8 & 71.0 & 0.812 & 0.803 & 3.025 & 0.172 & 19.2 & 80.8 & $<0.001$ & $0.60(0.54-0.66)$ \\
SCr-adm & 23.8 & 37.9 & 96.2 & 0.935 & 0.521 & 10.092 & 0.645 & 38.0 & 62.0 & $<0.001$ & $0.30(0.25-0.35)$ \\
\hline
\end{tabular}

$\mathrm{AKI}$, acute kidney injury; Cl, confidence interval; mSCr-base, measured SCr-base (within 3 months prior to admission); NLR, negative likelihood ratio; NPV, negative predictive value; PLR, positive likelihood ratio; PPV, positive predictive value; SCr, serum creatinine; SCr-adm, initial SCr value measured at pediatric intensive care unit admission.; SCr-eGFR, back-calculation of SCr values using age-adjusted normal reference value of eGFR; SCr-min, minimum SCr value measured within 7 days after pediatric intensive care unit admission; SCr-ref, normal reference SCr values adopted from the pediatric Sequential Organ Dysfunction Assessment score.

Table 4. Performance of the tested estimation methods in AKI staging and associated mortality relative to the use of baseline SCr levels measured preadmission

\begin{tabular}{|c|c|c|c|c|c|c|c|c|c|}
\hline \multirow{2}{*}{$\begin{array}{l}\text { Estimation } \\
\text { method }\end{array}$} & \multirow{2}{*}{ Category } & \multicolumn{4}{|c|}{ AKI stage } & \multirow{2}{*}{$\begin{array}{l}\text { Misclassification } \\
\text { rate }(\%)\end{array}$} & \multirow{2}{*}{$\begin{array}{c}\text { Percent } \\
\text { agreement (\%) }\end{array}$} & \multirow{2}{*}{$\begin{array}{l}\text { McNemar } \\
\text { test }\end{array}$} & \multirow{2}{*}{$\begin{array}{c}\text { Kappa statistics } \\
(95 \% \mathrm{Cl})\end{array}$} \\
\hline & & 0 & 1 & 2 & 3 & & & & \\
\hline mSCr-base & Staging (\%) & 41.2 & 23.4 & 15.8 & 19.6 & & & & \\
\hline \multirow[t]{2}{*}{ SCr-eGFR } & Staging (\%) & 80.1 & 6.5 & 2.5 & 10.8 & 49.4 & 50.8 & $<0.001$ & $0.22(0.18-0.26)$ \\
\hline & 28-Day mortality (\%) & 2.8 & 13.0 & 27.8 & 28.6 & 49.3 & 50.7 & $<0.001$ & $0.20(0.16-0.23)$ \\
\hline SCr-ref & Staging (\%) & 77.9 & 6.6 & 4.1 & 11.4 & 47.9 & 52.1 & $<0.001$ & $0.25(0.20-0.29)$ \\
\hline \multirow[t]{2}{*}{ SCr-min } & Staging (\%) & 36.5 & 32.8 & 17.2 & 13.5 & 23.9 & 76.1 & $<0.001$ & $0.51(0.46-0.56)$ \\
\hline & 28-Day mortality (\%) & 3.1 & 2.6 & 11.5 & 21.9 & 35.4 & 64.5 & $<0.001$ & $0.52(0.47-0.57)$ \\
\hline \multirow[t]{2}{*}{ SCr-adm } & Staging (\%) & 76.2 & 10.1 & 4.1 & 9.6 & 43.9 & 56.1 & $<0.001$ & $0.30(0.25-0.34)$ \\
\hline & 28-Day mortality (\%) & 2.4 & 12.5 & 24.1 & 29.4 & 44.9 & 55.1 & $<0.001$ & $0.32(0.27-0.37)$ \\
\hline
\end{tabular}

AKI, acute kidney injury; Cl, confidence interval; mSCr-base, measured SCr-base (within 3 months prior to admission); SCr, serum creatinine; SCr-adm, initial SCr value measured at pediatric intensive care unit admission; SCr-eGFR, back-calculation of SCr values using age-adjusted normal reference value of eGFR; SCr-min, minimum SCr value measured within 7 days after pediatric intensive care unit admission; SCr-ref, normal reference SCr values adopted from the pediatric Sequential Organ Dysfunction Assessment score.

\section{Acute kidney injury staging and associated 28-day mortal- ity rate}

Using mSCr-base, $23.4 \%, 15.8 \%$, and $19.6 \%$ of AKI cases were classified as stages 1, 2, and 3, respectively. In the comparison between $\mathrm{mSCr}$-base and the various eSCr-base values, SCr-min showed the best positive percent agreement of $76.1 \%$, with a kappa statistic of 0.510 (95\% CI, 0.463-0.557) for AKI staging (Table 4). The 28-day mortality rate showed a statistically significant tendency to increase along with the AKI stage calculated using mSCr-base and all the eSCr-base values $(\mathrm{p}<0.001)$. The 28-day mortality data sorted by AKI stage based on SCr-min showed the best agreement with those sorted by mSCr-base, with a positive percent agreement of $64.5 \%$ and a kappa statistic of 0.522 (95\% CI,
0.474-0.569).

\section{Discussion}

In this study, we evaluated four different methods for calculating a surrogate SCr-base value for critically ill pediatric patients and found overall poor agreement with mSCr-base.

No definite consensus-based diagnostic criteria for AKI are available for children; several standardized criteria have been adopted from those used in adults, including the pediatric Risk Injury Failure Loss End-stage Renal Disease (pRIFLE) criteria, the Acute Kidney Injury Network criteria, and the KDIGO criteria $[5,7,8,10,30]$. Most of those criteria require a baseline kidney function, represented by $\mathrm{mSCr}$ - 
base or GFR, to make an AKI diagnosis. However, mSCrbase is missing for up to $50 \%$ of patients, which makes the accurate evaluation of AKI and associated clinical outcomes difficult $[12,13,31]$. For patients without mSCr-base data, several previous studies in adults have evaluated different estimation methods $[13,15,16,18,32]$, but the pediatric and adult populations differ in several important, relevant ways. The most widely used approach in the adult population is the imputation method with the MDRD equation, which assumes an eGFR of $75 \mathrm{~mL} / \mathrm{min} / 1.73 \mathrm{~m}^{2}$ [13,16,33]. However, the MDRD equation is inappropriate in children because the normal reference eGFR differs by age, and the age- and growth-dependent reference ranges for SCr and eGFR values in pediatric patients are wide $[21,23,34,35]$. In this study, we used the age-adjusted normal reference values for eGFR and SCr [21-24] in our SCr-eGFR and SCr-ref, calculations, respectively. Nonetheless, SCr-eGFR and SCr-ref showed poor agreement with mSCr-base. The finding that mSCrbase was smaller than both SCr-eGFR and SCr-ref can be explained using various factors that depend on the conditions of individual patients. Most of the patients included in this study had underlying disorders, most commonly cardiac problems. In addition, $27.0 \%$ had severe malnourishment, which could be related to decreased muscle mass. Consequently, assuming that the renal function of critically ill pediatric patients admitted to a PICU is equivalent to that of "normal healthy" children may be inappropriate.

Although the definition of mSCr-base used here, an SCr value measured within 3 months before hospitalization, is the most widely accepted, the use of mean, most recent, or nadir outpatient SCr values at time intervals of up to 730 days before hospitalization has been suggested in adult population studies $[11,15,36]$. However, in children, the normal reference values can change significantly over time, so using older data could lead to AKI evaluation inaccuracies. Furthermore, the use of data from $>3$ months of hospitalization could mistakenly suggest chronic renal failure. Therefore, we used SCr values measured within 3 months before hospitalization as mSCr-base.

SCr-min was another eSCr-base method used in this study. Although a strong consensus exists that the lowest SCr value should be used for the SCr-min calculation, the duration within which the lowest SCr value is determined varies among the relevant studies, ranging from the whole hospitalization period to 2 weeks or 3 days after PICU ad- mission $[11,13,16,17,31,32,37]$. In this study, SCr-min was defined as the lowest SCr value measured within 7 days after PICU admission for the following reasons. First, that time period is consistent with the diagnostic criteria provided by pRIFLE and KDIGO. Second, from a clinical standpoint, a longer time window could impede the prompt evaluation and management of AKI. Third, the condition of pediatric patients admitted to a PICU with temporary AKI caused by dehydration or acute deterioration, often improves dramatically with treatment.

Among the eSCr-base calculation methods tested here, SCr-min showed the best agreement with $\mathrm{mSCr}$-base (i.e., the actual SCr-based value), with the largest ICC, smallest bias, and best percent agreement in AKI diagnosis, staging, and the associated 28-day mortality rate. However, it slightly overestimated the incidence of AKI. The eSCr-base values calculated using the other methods underestimated the incidence of AKI; thus, the diagnosis and staging of AKI based on those calculations were inaccurate, with large misclassification rates.

In adult populations, researchers have suggested several approaches for SCr-base imputation and reported various degrees of agreement between mSCr-base and their eSCr-base calculations. However, the best technique to use remains controversial; no definite universal baseline SCr or eSCr-base method has reached consensus-level support. In addition, some investigators have expressed significant disagreement consistent with our results and suggested the use of recorded SCr values whenever possible [15,32,38].

Critically ill patients in intensive care unit settings differ from the normal healthy population in many important ways that can affect their SCr values, renal function, and AKI evaluation, such as chronic illness, impaired nutritional status, decreased muscle mass, fluid imbalance, and the use of various medications [31]. Likewise, individual characteristics and clinical situations might need to be considered to accurately estimate SCr-base and achieve good agreement with the actual mSCr-base. In this study, SCr-min showed the best agreement with mSCr-base, which suggests that considering individual traits might provide a better estimate of a patient's steady-state than using a value assumed to represent "normal healthy" children.

On the other hand, SCr itself has several drawbacks as a marker of renal function. It is affected by age, sex, ethnicity, dietary factors, and muscle mass. In addition, it is actively 
secreted by the proximal tubule, leading to an overestimation of GFR. There is also a time lag between significant renal injury and the elevation of SCr levels. Cystatin C was recently introduced as an alternative endogenous marker of renal function because it is freely filtered by the renal glomerulus, does not have non-renal elimination or glomerular secretion, and is independent of muscle mass. Consequently, cystatin $\mathrm{C}$ measurement could provide some advantages in evaluating AKI, especially in critically ill children $[39,40]$. Large-scale, well-designed studies of pediatric patients are needed to develop pediatric-specific definitions, diagnostic tools, and staging criteria for AKI that use proper markers, including cystatin C.

This study has some limitations. First, it was a single-center retrospective study. Due to the characteristics of the study population, the results might have limited generalizability. Second, in back calculating SCr from eGFR, we used the original Schwartz equation (instead of the revised bedside Schwartz equation) because it was derived from SCr measured using the Jaffe method, which is used to measure $\mathrm{SCr}$ in our institution. However, the kinetic Jaffe assay can be affected by factors such as albumin, glucose, and bilirubin in ways that can result in the overestimation of $\mathrm{SCr}$ $[25,27,41]$.

Third, mSCr-base was defined as the lowest value within 3 months before PICU admission. All the SCr values included were measured regardless of the patients' status and whether they were drawn in the emergency room or at outpatient clinics. Those values might not fully represent the patients' best normal condition. Fourth, only SCr-based criteria were used for AKI diagnosis and classification. However, a recent report emphasizes that changes in urine output can be helpful for detecting AKI that might be missed when using only SCr criteria [2].

Despite those limitations, this study has several strengths. Its main advantage is that, to the best of our knowledge, it is the first to evaluate different eSCr-base methods in critically ill pediatric patients. In addition to considering methods widely studied and used in adult populations, we examined the characteristics of the children and used age-adjusted normal reference SCr and eGFR values. We further evaluated not only the agreement between each eSCr-base and mSCr-base, but also the clinical performance of each eSCr-base and mSCr-base in AKI diagnosis and staging to determine which method could be most useful in clinical practice. Because this was a single-center study, unified diagnosis and staging using the same criteria and general intensive care were applied consistently throughout the study period, which reduced the number of confounding factors.

In conclusion, the diagnosis and staging of AKI are greatly affected by the SCr-base value used; therefore, the careful selection of an appropriate SCr-base is important. In this study, SCr-min showed the best agreement with mSCr-base in both the actual SCr value calculated and the diagnosis and staging of AKI. However, further large-scale studies are required to establish definite, accurate diagnostic and staging criteria for AKI in critically ill pediatric patients.

\section{Conflicts of interest}

No potential conflicts of interest relevant to this article are reported.

\section{Acknowledgments}

We thank all our colleagues involved in the care of COVID-19 patients for their intellectual generosity. This study did not receive any specific grants from funding agencies in the public, commercial, or not-for-profit sectors.

\section{Authors' contributions}

Conceptualization: SJP, WKJ

Data curation: YJL, WKJ

Formal analysis: WKJ

Intellectual contributions: YSP

Writing-original draft: YJL, WKJ

Writing-review \& editing: All authors

All authors read and approved the final manuscript.

\section{ORCID}

Yeon Joo Lee, https://orcid.org/0000-0003-4092-8009

Young Seo Park, https://orcid.org/0000-0001-7653-2063

Seong Jong Park, https://orcid.org/0000-0003-0250-2381

Won Kyoung Jhang, https://orcid.org/0000-0003-2309-0494

\section{References}

1. Alobaidi R, Morgan C, Goldstein SL, Bagshaw SM. Popula- 
tion-based epidemiology and outcomes of acute kidney injury in critically ill children. Pediatr Crit Care Med 2020;21:82-91.

2. Kaddourah A, Basu RK, Goldstein SL, Sutherland SM; Assessment of Worldwide Acute Kidney Injury, Renal Angina and, Epidemiology (AWARE). Oliguria and acute kidney injury in critically ill children: implications for diagnosis and outcomes. Pediatr Crit Care Med 2019;20:332-339.

3. Wang L, McGregor TL, Jones DP, et al. Electronic health record-based predictive models for acute kidney injury screening in pediatric inpatients. Pediatr Res 2017;82:465-473.

4. Gist KM, Selewski DT, Brinton J, Menon S, Goldstein SL, Basu RK. Assessment of the independent and synergistic effects of fluid overload and acute kidney injury on outcomes of critically ill children. Pediatr Crit Care Med 2020;21:170-177.

5. Akcan-Arikan A, Zappitelli M, Loftis LL, Washburn KK, Jefferson LS, Goldstein SL. Modified RIFLE criteria in critically ill children with acute kidney injury. Kidney Int 2007;71:1028-1035.

6. Mehta RL, Kellum JA, Shah SV, et al. Acute Kidney Injury Network: report of an initiative to improve outcomes in acute kidney injury. Crit Care 2007;11:R31.

7. Kellum JA, Levin N, Bouman C, Lameire N. Developing a consensus classification system for acute renal failure. Curr Opin Crit Care 2002;8:509-514.

8. Sutherland SM, Byrnes JJ, Kothari M, et al. AKI in hospitalized children: comparing the pRIFLE, AKIN, and KDIGO definitions. Clin J Am Soc Nephrol 2015;10:554-561.

9. Kellum JA, Lameire N; KDIGO AKI Guideline Work Group. Diagnosis, evaluation, and management of acute kidney injury: a KDIGO summary (Part 1). Crit Care 2013;17:204.

10. Khwaja A. KDIGO clinical practice guidelines for acute kidney injury. Nephron Clin Pract2012;120:c179-c184.

11. Lewington A, Kanagasundaram S. Renal Association Clinical Practice Guidelines on acute kidney injury. Nephron Clin Pract 2011;118 Suppl 1:c349-c390.

12. Alkandari O, Eddington KA, Hyder A, et al. Acute kidney injury is an independent risk factor for pediatric intensive care unit mortality, longer length of stay and prolonged mechanical ventilation in critically ill children: a two-center retrospective cohort study. Crit Care 2011;15:R146.

13. Bernier-Jean A, Beaubien-Souligny W, Goupil R, et al. Diagnosis and outcomes of acute kidney injury using surrogate and imputation methods for missing preadmission creatinine values. BMC Nephrol 2017;18:141.

14. Hoste EA, Clermont G, Kersten A, et al. RIFLE criteria for acute kidney injury are associated with hospital mortality in critically ill patients: a cohort analysis. Crit Care 2006;10:R73.

15. Siew ED, Matheny ME. Choice of reference serum creatinine in defining acute kidney injury. Nephron 2015;131:107-112.

16. Lang XB, Yang Y, Yang JR, et al. Comparison of three methods estimating baseline creatinine for acute kidney injury in hospitalized patients: a multicentre survey in third-level urban hospitals of China. Kidney Blood Press Res 2018;43:125-133.

17. Pickering JW, Endre ZH. Back-calculating baseline creatinine with MDRD misclassifies acute kidney injury in the intensive care unit. Clin J Am Soc Nephrol 2010;5:1165-1173.

18. Kork F, Balzer F, Krannich A, et al. Back-calculating baseline creatinine overestimates prevalence of acute kidney injury with poor sensitivity. Acta Physiol (Oxf) 2017;219:613-624.

19. Liu KD, Hsu CY, Yang J, et al. Acute kidney injury ascertainment is affected by the use of first inpatient versus outpatient baseline serum creatinine. Kidney Int Rep 2017;3:211-215.

20. Wald R. Predicting baseline creatinine in hospitalized patients. Clin J Am Soc Nephrol 2012;7:697-699.

21. Pottel H. Measuring and estimating glomerular filtration rate in children. Pediatr Nephrol 2017;32:249-263.

22. Schwartz GJ, Work DF. Measurement and estimation of GFR in children and adolescents. Clin J Am Soc Nephrol 2009;4:18321843.

23. Pottel H, Mottaghy FM, Zaman Z, Martens F. On the relationship between glomerular filtration rate and serum creatinine in children. Pediatr Nephrol 2010;25:927-934.

24. Matics TJ, Sanchez-Pinto LN. Adaptation and validation of a pediatric sequential organ failure assessment score and evaluation of the Sepsis-3 definitions in critically ill children. JAMA Pediatr 2017;171:e172352.

25. Hoste L, Deiteren K, Pottel H, Callewaert N, Martens F. Routine serum creatinine measurements: how well do we perform? BMC Nephrol 2015;16:21.

26. Delanghe JR, Speeckaert MM. Creatinine determination according to Jaffe-what does it stand for? NDT Plus 2011;4:83-86.

27. Drion I, Cobbaert C, Groenier KH, et al. Clinical evaluation of analytical variations in serum creatinine measurements: why laboratories should abandon Jaffe techniques. BMC Nephrol 2012;13:133.

28. Muhari-Stark E, Burckart GJ. Glomerular filtration rate estimation formulas for pediatric and neonatal use. J Pediatr Pharmacol Ther 2018;23:424-431.

29. Pollack MM, Patel KM, Ruttimann UE. PRISM III: an updated Pediatric Risk of Mortality score. Crit Care Med 1996;24:743-752.

30. Nahum E, Kadmon G, Kaplan E, et al. Prevalence of acute kidney 
injury after liver transplantation in children: comparison of the pRIFLE, AKIN, and KDIGO criteria using corrected serum creatinine. J Crit Care 2019;50:275-279.

31. De Rosa S, Samoni S, Ronco C. Creatinine-based definitions: from baseline creatinine to serum creatinine adjustment in intensive care. Crit Care 2016;20:69.

32. Siew ED, Matheny ME, Ikizler TA, et al. Commonly used surrogates for baseline renal function affect the classification and prognosis of acute kidney injury. Kidney Int 2010;77:536-542.

33. Hoste EA, Bagshaw SM, Bellomo R, et al. Epidemiology of acute kidney injury in critically ill patients: the multinational AKI-EPI study. Intensive Care Med 2015;41:1411-1423.

34. Pottel H, Vrydags N, Mahieu B, Vandewynckele E, Croes K, Martens F. Establishing age/sex related serum creatinine reference intervals from hospital laboratory data based on different statistical methods. Clin Chim Acta 2008;396:49-55.

35. Hawkins RC. Differences in serum creatinine concentration between Caucasians, Chinese, Indians and Malays. Clin Chim Acta 2010;411:1393.
36. Siew ED, Ikizler TA, Matheny ME, et al. Estimating baseline kidney function in hospitalized patients with impaired kidney function. Clin J Am Soc Nephrol 2012;7:712-719.

37. Broce JC, Price LL, Liangos O, Uhlig K, Jaber BL. Hospital-acquired acute kidney injury: an analysis of nadir-to-peak serum creatinine increments stratified by baseline estimated GFR. Clin J Am Soc Nephrol 2011;6:1556-1565.

38. Gaião S, Cruz DN. Baseline creatinine to define acute kidney injury: is there any consensus? Nephrol Dial Transplant 2010;25:3812-3814.

39. Filler G, Huang SH, Yasin A. The usefulness of cystatin C and related formulae in pediatrics. Clin Chem Lab Med 2012;50:20812091.

40. Safdar OY, Shalaby M, Khathlan N, et al. Serum cystatin is a useful marker for the diagnosis of acute kidney injury in critically ill children: prospective cohort study. BMC Nephrol 2016;17:130.

41. Panteghini M; IFCC Scientific Division. Enzymatic assays for creatinine: time for action. Clin Chem Lab Med 2008;46:567-572. 